\title{
Three-Dimensional Velocity Obstacle Method for UAV Deconflicting Maneuvers
}

\author{
Yazdi I. Jenie* Erik-Jan van Kampen ${ }^{\dagger}$ Coen C. de Visser ${ }^{\ddagger}$ Joost Ellerbroek $^{\S}$ Jacco M. Hoekstra $^{\llbracket}$ \\ Control and Simulation Section, Faculty of Aerospace Engineering, Delft University of Technology
}

\begin{abstract}
Autonomous systems are required in order to enable UAVs to conduct self-separation and collision avoidance, especially for flights within the civil airspace system. A method called the Velocity Obstacle Method can provide the necessary situational awareness for UAVs in a dynamic environment, and can help to generate a deconflicting maneuver. This paper focuses on the assessment of the Velocity Obstacle Method application and its ability to resolve various conflict situations in three dimensional space. This assessment results in a redefinition of the criteria of avoidance. A novel technique is introduced to support the avoidance decision, by representing the conflict situation in various avoidanceplanes. Several new definitions to support the method are introduced. This method is then implemented in three-dimensional simulations for UAVs in cases of conflict, in which more than one option of resolution is provided.
\end{abstract}

\section{Nomenclature}

$\phi \quad$ Dihedral angle of the avoidance-plane from the XY-plane, [-]

$\theta_{a z} \quad$ Azimuth angle of the Velocity Obstacle Cone, [-]

$\theta_{a z} \quad$ Elevation angle of the Velocity Obstacle Cone, [-]

$\theta_{P_{\phi}} \quad$ Dihedral angle between $B_{v o}$ and $P_{\phi},[\mathrm{m}]$

$\theta_{v o} \quad$ Opening angle of the Velocity Obstacle Cone, $[\mathrm{m}]$

$A_{v o} \quad$ Apex of the Velocity Obstacle Cone, [m]

$B_{c c} \quad$ Collision Cone base-circle, [-]

$B_{v o} \quad$ Base-circle of the Velocity Obstacle Cone, [m]

$C_{v o} \quad$ Center of the base of the Velocity Obstacle Cone, $[\mathrm{m}]$

$C C \quad$ Collision cone set of obstacle, $[\mathrm{m} / \mathrm{s}]$

$d_{\text {avo }} \quad$ Distance of avoidance starting point from the obstacle, $[\mathrm{m}]$

$D_{o i} \quad$ Vector line from the ownship $A_{o}$ to obstacle, [m]

$d_{o i} \quad$ Distance of the own-ship from the obstacle, [m]

$d_{v o} \quad$ Length of the axis of symmetry of Velocity Obstacle Cone, $[\mathrm{m}]$

$D I V \quad$ Diverging Zone set, $\{[\mathrm{m} / \mathrm{s}] ;[\mathrm{m} / \mathrm{s}]\}$

$G_{v o} \quad$ Generating line of the Velocity Obstacle Cone, [m]

$H_{v o} \quad$ Axis of symmetry of the Velocity Obstacle Cone, [m]

$l_{v d} \quad V O-D I V$ intersection line, $\{[\mathrm{m}] ;[\mathrm{m}] ;[\mathrm{m}]\}$

$P_{\phi} \quad$ Avoidance Plane at the angle $\phi,[-]$

$r_{v o} \quad$ Radius of the base-circle of Velocity Obstacle Cone, [m]

$R V \quad$ Reachable Velocity set, $\{[\mathrm{m} / \mathrm{s}] ;[\mathrm{m} / \mathrm{s}]\}$

$S_{p z} \quad$ Protected Zone, $\{[\mathrm{m}] ;[\mathrm{m}]\}$

$S_{V O_{P}}$ Area of the Velocity Obstacle on the respective $P_{\phi},\left[\mathrm{m}^{2}\right]$

$V_{o}, V_{i} \quad$ Velocity of $A_{o}$, Velocity of $A_{i},[\mathrm{~m}]$

*PhD Student, Faculty of Aerospace Engineering, Delft University of Technology, 2629HS, Delft, The Netherlands

${ }^{\dagger}$ Assistant Professor, Faculty of Aerospace Engineering, Delft University of Technology, 2629HS, Delft, The Netherlands

¥Assistant Professor, Faculty of Aerospace Engineering, Delft University of Technology, 2629HS, Delft, The Netherlands

$\S$ Assistant Professor, Faculty of Aerospace Engineering, Delft University of Technology, 2629HS, Delft, The Netherlands

\Professor, Faculty of Aerospace Engineering, Delft University of Technology, 2629HS, Delft, The Netherlands 
$V_{R} \quad$ Relative velocity between two vehicles $[\mathrm{m} / \mathrm{s}]$

$V_{\text {avo }} \quad$ Avoidance velocity, $[\mathrm{m} / \mathrm{s}]$

$V_{\text {avo }}^{\min } \quad$ Closest point for avoidance velocity, $[\mathrm{m} / \mathrm{s}]$

$V O \quad$ Velocity Obstacle set, $\{[\mathrm{m} / \mathrm{s}] ;[\mathrm{m} / \mathrm{s}]\}$

$X_{o}, X_{i}$ Position of the own-ship, Position of the obstacle, [m]

\section{Introduction}

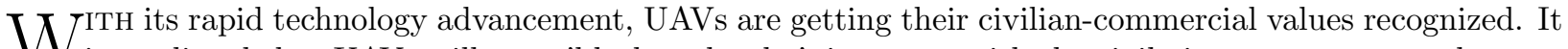
W is predicted that UAVs will soon 'blacken the sky', integrate with the civil airspace system, and create a whole new type of traffic. Ref 1 gives several predictions on the usage of UAVs for civilians purposes. Once this integration is allowed, the expansion of UAVs into the airspace is inevitable; Many manufacturers and operators will race to exploit them, such that managing their air traffic will be a major concern. Since the UAVs will range widely in type and mission, dependence on a common ground station and predefined airways, like in manned-flight, is impractical. Ref.2 suggests the use of the self separation (or free flight) concept $^{3,4}$ that allows free routing and optimization of each vehicle separately.

The self-separation concept switches the task for separation keeping as well as collision avoidance to each UAV, instead of a ground control station. This task, however, will be difficult to be handle manually, due to the operators lack of situational awareness. The task is suggested to be handled autonomously. ${ }^{5}$ Hence, research for UAVs is currently focused more on an autonomous Conflict Detection and Resolution (CD\&R) system. Ref.2 presents a method for such a system that generates a deconflict maneuver ${ }^{5,2}$ that is guided by common rules of the air. The method is called the Selective Velocity Obstacle (SVO) Method and it is an extension of the Velocity Obstacle Method (VO-method). The SVO method is able to generate an avoidance path for each UAV separately and to resolve every conflict that occurs, despite being heterogeneous.

Those demonstrations however, are restricted to a two-dimensional scenario, where it is assumed that the UAVs fly at the same altitude. This makes the SVO inadequate for UAVs that can use the full three dimensional space. In fact, the VO-method itself has been developed mainly in two dimensions since the first works on it are for land-robots. Currently there are only a few publications that mention application of the VO-method on three dimensional cases. Among them are the works of Ref.6,7, that uses the VO-method to support conflict visualization for a pilot in a non autonomous manned-flight.

This paper focuses on the assessment of the Velocity Obstacle method in three dimensional cases of UAV conflicts, and on how a resolution strategy can be decided upon. The VO-method is explained in a similar manner as Ref.2, elaborated by its criteria, algorithm, and strategy. The main goal is to modify the former VO-method for the three dimensional space, especially for UAVs applications. The modification is focused on the three main VO-method sets, i.e. the Velocity Obstacle, $V O$, the Diverging Zone, DIV, and the Reachable Velocity, $R V$. A novel technique is introduced that represents the conflict situation in various avoidance-planes. These avoidance-planes will help the autonomous system to decide which avoidance is the fastest, or which has the least risk. The avoidance maneuver will consider the deconflicting maneuver, in which the avoidance is conducted with as small of a deviation from the original path as possible. Since it is still preliminary, any uncertainties in the flight data are neglected.

This paper is structured as follows. After this introduction, the second section discusses the concept of the original Velocity Obstacle Method for deconflicting maneuver, as the basis of this research. This is the VO-method for two dimensional encounter cases. Section III explains the VO-method set definitions in three-dimensional space. The concept of avoidance-plane is introduced specifically in the following Section IV along with several new deifnition and calculation techniques to help the decision making for avoidance. Section V presents the implementation of the three-dimensional VO-method in simulations. Here, several encounter cases, as well as choice of avoidance are simulated and discussed. Section VI ends the paper with several concluding remarks.

\section{Velocity Obstacle Method}

This section explains briefly the Velocity Obstacle method ${ }^{8,9,10,11,12,13,6,7,14,2}$ commonly used in the two dimensional conflict scenario between two vehicles. The explanation will take the same approach that Ref.2 used, which uses a division into the parts: VO-method criteria, algorithm, and strategy. The objective of the VO-method in this paper is to generate a deconflicting maneuver that can avoid the protected-zone, 
which, in two-dimensions, is the circle $S_{p z}$, as shown in Figure 1.

\section{A. Criteria}

Consider an encounter of two UAVs, the own-ship and the obstacle, flying on the same altitude, shown in a top-down point of view in Figure 1.

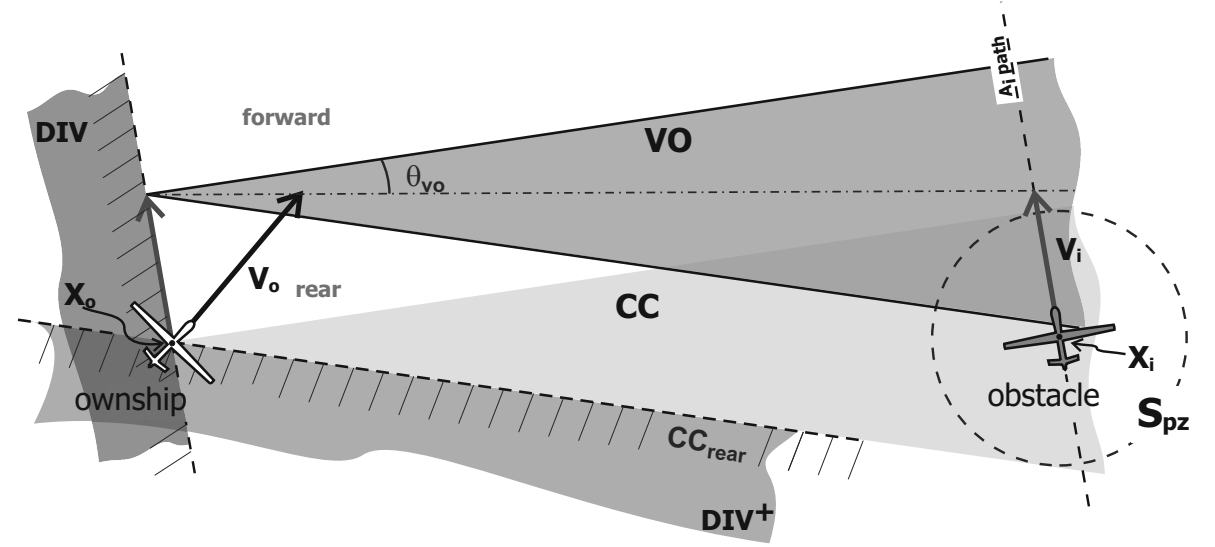

Figure 1. Two-dimensional Velocity Obstacle method. Definitions of the Collision Cone $C C$, the Velocity Obstacle set $V O$, and the new definition of the $D I V$ set

The circle around the obstacle is the protected-zone circle, $S_{p z}$, that should not be violated by the ownship; violation would mean that the ownship and the obstacle collide. Both vehicles are moving with a constant velocity $V_{o}$ and $V_{i}$, respectively, in a horizontal plane. With this setup, the collision cone $C C$ can be defined as the set that collects all relative velocities $V_{R}$ whose extension would intersect the $S_{p z}$ circle. This means that $C C$ is the area within two tangent lines of $S_{p z}$ from the point $X_{o}$, the current position of the ownship. Hence, every combination of velocity $V_{o}$ and $V_{i}$ that produces a relative velocity $V_{R}$ that is included in the collision cone $C C$ will make the two vehicles collide, sometime in the future.

The velocity obstacle set, $V O$, is produced by translating the $C C$ along the obstacle's velocity $V_{i}$. The VO-method also defines a diverging set, $D I V$, in which every $V_{o}$ that is diverging from the obstacle's flight path is collected. The $D I V$ is defined as one of the half-planes separated by the $V_{i}$ line that does not contain the $V O$ set. This definition, however, is found inadequate since it does not consider the part of the obstacle's flight path that has been passed. Ref.14 noted several cases of late restoring in the avoidance, since even after the obstacle is cleared, the ownship has to stay in maintain mode until it crosses the flight path. This paper introduce a new definition of the diverging set DIV as a collection of velocities that diverge from the obstacle $A_{i}$ and its path ahead, excluding the part of the path that is already passed. The rear edge of $C C$ is the direct connection for this, producing a $D I V$ as a summation of the half-plane by $V_{i}$ (the original definition of $D I V$ ) and the $C C$ rear area (the $D I V^{+}$), as shown in Figure 1.

The VO-method uses the sets to describe an encounter situation in the VO criteria as follows:

$C_{1}^{v o}$ : An encounter between the ownship and an obstacle- $i$ is imminent if, and only if, $d_{o i} \leq d_{a v o}$.

$C_{2}^{v o}$ : The ownship will violate the $S_{p z}$ of an obstacle- $i$ sometime in the future if, and only if, $V_{o} \in V O_{o i}$.

$C_{3}^{v o}$ : The ownship is diverging an obstacle- $i$ if, and only if, $V_{o} \in D I V_{o i}$.

The first criterion, $C_{1}^{v o}$, is an additional criteria to decide whether the encounter between two vehicles is imminent or not, based on the distance between them, $d_{o i}$, and the choice of distance to start avoidance $d_{\text {avo }}$. The SVO presented in Ref.2 presents five additional criteria to further determine the type of encounter, and assigns right-of-way along with it. These additional criteria, however, are not discussed in this paper.

\section{B. Algorithm}


In this paper, the VO-method uses three modes: avoid, maintain, and restore. This approach is similar with Ref.14 with a change in the first mode; the avoid-mode is used to generalize the turn-mode used for avoidance. These three modes are used especially to generate a deconflicting maneuver, a maneuver that can resolve conflict while keeping the deviation as small as possible. ${ }^{5,14,2}$ The maintainmode is especially defined to eliminate the oscillation problem that occurs in the basic VO-method. ${ }^{15}$

The algorithm, shown in figure 2, works as follows: When an ownship, $A_{o}$, encounters an obstacle, $A_{i}$, the autonomous CD\&R system analyzes the situation by the evaluation of the criteria. If the encounter is not imminent, or if $V_{o}$, the velocity of $A_{o}$, is diverging from the obstacle flight path, $\neg C_{1}^{v o} \vee C_{3}^{v o}$, then $A_{o}$ can continue its original mission, or from the $\mathrm{VO}$ point of view, stay in its restoremode. If the encounter is imminent and $A_{o}$ is not diverging, $C_{1}^{v o} \wedge$

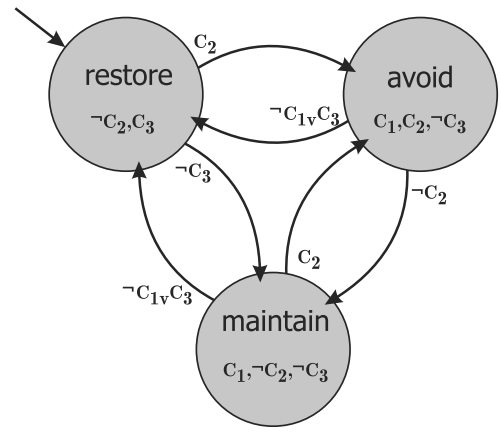

Figure 2. State transition diagram of the Velocity Obstacle method algorithm $\neg C_{3}^{v o}$, then the system needs to analyze the inclusion of $V_{o}$ inside the $V O$. If $V_{o}$ is included in $v o, C_{2}^{v o}$, then $A_{o}$ should change to avoid-mode, if not, $\neg C_{2}^{v o}, A_{o}$ has to change to maintain-mode. The combination of the algorithm and the VO-method criteria result in a precise and deterministic action for a vehicle to either avoid, maintain, or restore its velocity, in a dynamic encounter. The decision can be made in an instance, without requiring any prediction of obstacle movement.

\section{Strategy}

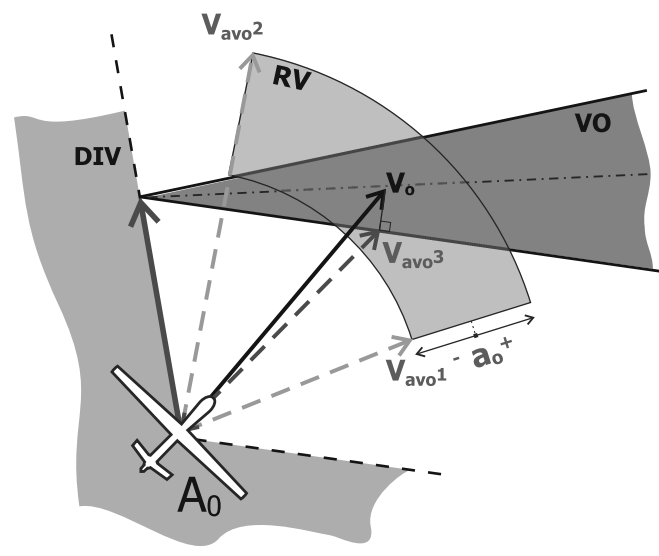

Figure 3. The Reachable Velocity Set that contains every velocity $A_{o}$ can choose in its avoidance within a time-step, e.g. $V_{a v o^{1}}$, $V_{a v o^{2}}$, and $V_{a v o^{3}}$

The strategy of the VO-method describes the option of changing velocity, within a mode selected by the UAV. The maintainmode certainly only has one option, to keep the current velocity. In restore-mode the control of the own-ship is given back to the original control system to continue its mission. Commonly in many research of the $\mathrm{VO}$-method, the restore-mode points the vehicle back to its original goal point, which is also done in this paper. Unlike the maintain and restore modes, the strategy in the avoid-mode has several variations throughout VO-research.

The strategy in the avoid-mode can be described using the reachable velocity set, $R V$, a collection of avoidance velocities, $V_{\text {avo }}$ an own-ship can choose within a time-step. Ref.14 limits the avoidance maneuver into turning only, to make it more suitable in airborne vehicle application, and thus describes the $R V$ as an arc. Relaxing this limitation, the research in this paper will use an fan-shaped $R V$ instead, which is still dominated by the turning maneuver, but has options to slow down or speed up, as shown in Figure 3. The strategy for avoidance is to choose the closest new velocity within the $R V$ set, that is not intersecting with the $V O$. In Figure 3, the closest new velocity for avoidance is shown by the velocity $V_{a v o^{3}}$, which is simply the intersection point of a line from $V_{o}$, perpendicular with $V O$ 's rear edge.

\section{Three Dimensional Velocity Obstacle}

The previous section presented the conventional use of the velocity obstacle in two-dimensional space, divided into three parts, the criteria, algorithm, and strategy. These three parts remain the same in threedimensional space. The sets used, however, are redefined. They include the Velocity Obstacle set, $V O$, the Diverging zone set, $D I V$, and the Reachable Velocity set, $R V$. 


\section{A. Velocity Obstacle Set in Three Dimension}

The work of Ref. 6 and Ref.7 are two examples that extend the use of VO in three dimensions. Those works use a disk, or cylinder, to define the protected zone. The collision cone, and hence the VO, is shaped by taking the outer most lines that connect $X_{o}$ with the disk. The disk shape is chosen since the work of Ref.6 and Ref.7 is used for separation assurance, with distances more than 50 NM. Vertical separation is considering the predefined traffic. Manned-flights in those distances are usually mostly horizontal. On the other hand, the three-dimensional VO-method in this paper aims to provide a method of collision avoidance for UAVs, that are expected to fill the sky with various mission and types. The flight path of each UAV is assumed to go in every direction. Therefore, this paper takes a different approach and uses a sphere, $S_{p z}$, to define the protected zone in space, as shown in Figure 4.

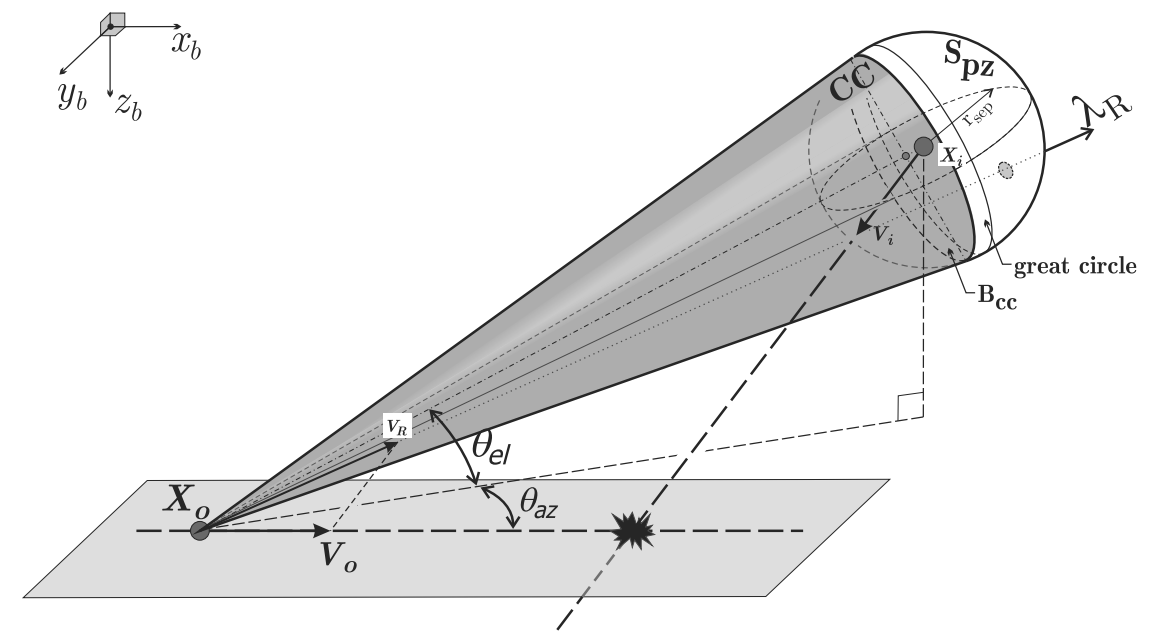

(a)

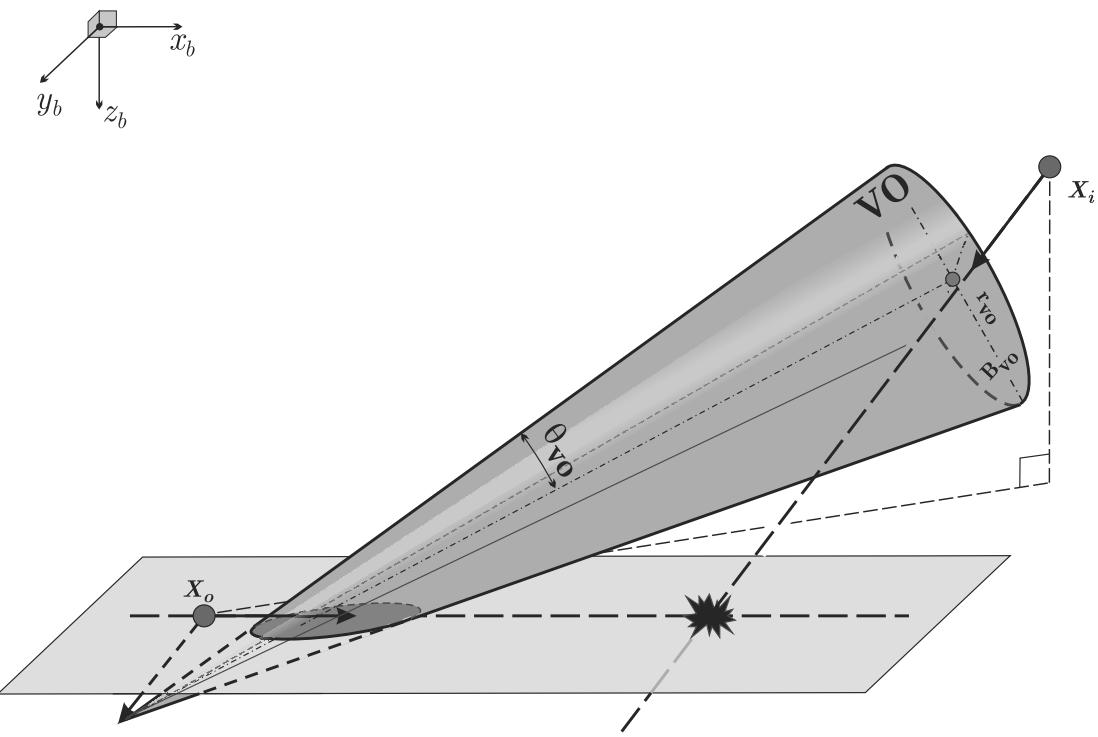

(b)

Figure 4. Three dimensional velocity obstacle set definition. (a) the Collision Cone $C C$, (b) Translated $C C$ cone to the Velocity Obstacle set $V O$

Figure 4-a shows the collision cone, $C C$, as a real right-cone with as generating lines the tangent lines of the $S_{p z}$ sphere from the point $X_{o}$. It can be observed that the $C C$ cone is a collection of every relative velocity $V_{R}$ in three-dimensions, that can extend through the $S_{p z}$ sphere, shown for example as the $\lambda_{R}$ line 
in figure 4-a. With $D_{o i}$ defined as the vector connecting $X_{o}$ and $X_{i}$, let $B_{c c}$ be the area perpendicular with $D_{o i}$ as the base of $C C$. Notice that $B_{c c}$ does not cut through $S_{p z}$ on its center. In fact, since it contains all the tangent points of $S_{p z}$ from $X_{o}, B_{c c}$ will always be smaller than then $S_{p z}$ circle.

Similar to the two-dimensional case, the $V O$ is obtained by translating the $C C$ along $V_{i}$, as shown in Figure 4-b. The base circle of the $V O$ cone is defined as $B_{v o}$, with radius of $r_{v} o$ and distance from $V O$ apex as $d_{v o}$, presented in equation (1) and equation (2), respectively. The $V O$ cone opening angle also describes the shape of the cone, and is given in equation (3)

$$
\begin{gathered}
r_{v o}=r_{p z} \frac{\sqrt{d_{o i}^{2}-r_{p z}^{2}}}{d_{o i}} \\
d_{v o}=\frac{d_{o i}^{2}-r_{p z}^{2}}{d_{o i}} \\
\theta_{v o}=\arctan \frac{r_{v o}}{d_{v o}}
\end{gathered}
$$

In this setup, the two vehicles will collide some time in the future if $V_{o} \in V O$, assuming both velocities are constant. Ref. 2 uses the edges of $V O$ to determine whether $V_{o}$ is included within. One alternative to determine whether the $V_{o}$ point is inside the three dimensional cone $V O$ is by transforming the $V_{o}$ point to the cone's frame of reference, where the apex is the origin, and the axis is the x-axis, positive to the cone base $B_{v o}$, as presented in equation (4).

$$
\left[\begin{array}{c}
V_{o x}^{\prime} \\
V_{o y}^{\prime} \\
V_{o z}^{\prime}
\end{array}\right]=\left[\begin{array}{ccc}
\cos \theta_{a z} \cos \theta_{e l} & \sin \theta_{a z} & \cos \theta_{a z} \sin \theta_{e l} \\
-\sin \theta_{a z} \cos \theta_{e l} & \cos \theta_{a z} & -\sin \theta_{a z} \sin \theta_{e l} \\
-\sin \theta_{e l} & 0 & \cos \theta_{e l}
\end{array}\right]\left[\begin{array}{c}
V_{o x}-V_{i x} \\
V_{o y}-V_{i y} \\
V_{o z}-V_{i z}
\end{array}\right]
$$

Then, the condition $V_{o} \in V O$ is met if, and only if, equation (5) is fulfilled.

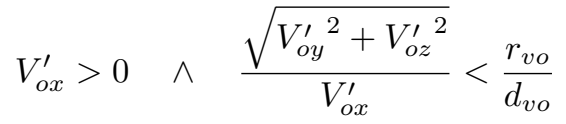

\section{B. Diverging Velocity Set in Three Dimension}

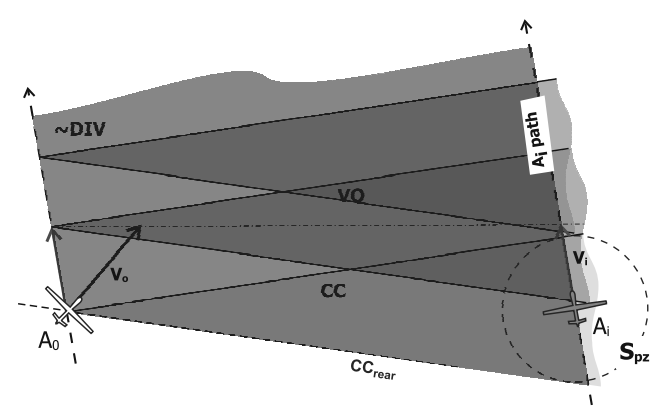

(a)

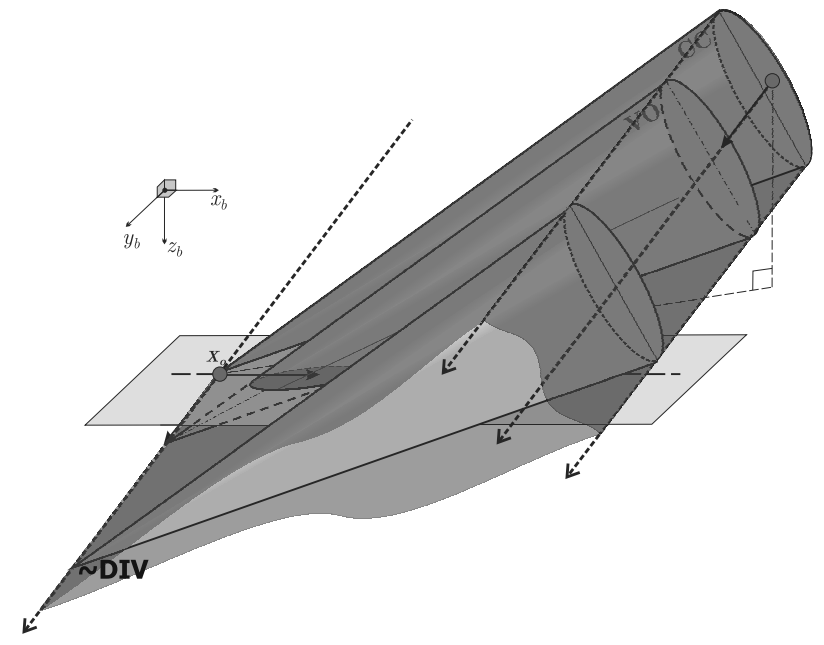

(b)

Figure 5. The non-diverging set, $\neg D I V$, generation by sweeping the plane/space with the collision cone along the $V_{i}$, starting from the current $A_{o}$ position. This is in order to indirectly define the Diverging Zone $D I V$ (a) in two-dimensions; (b) In three-dimensions 
The definition of diverging area in three dimensions, the diverging zone, is not as straightforward as the $V O$. This zone is needed to define where the $A_{o}$ can safely change to the restore-mode. The diverging zone $D I V$ can be viewed as a collection of velocities $V_{o}$ that make $A_{o}$ diverge from the obstacle and its path ahead. Figure 5-a shows an area in a two-dimensional situation that is generated by sweeping the Collision Cone $C C$, from $X_{o}$, along the obstacle velocity, $V_{i}$. The area that is not include is therefore the diverging area, $D I V$, as defined in the previous section. Using a similar approach, Figure 5-b shows the generation of the zone of not-diverging, $\neg D I V$, by sweeping the three dimensional space with the collision cone from the $X_{o}$ along $V_{i}$. The whole space that is outside this prism-zone is therefore the diverging zone, $D I V$.

With this definition, the three-dimensional $D I V$ seems to dominate the space, suggesting that the risk of conflict in three-dimensional space is much less than in the two-dimensional plane. It can also be observed that there exist parts of the $V O$ cone that touch directly with the diverging zone. These parts are the two opposite generating-lines of the $V O$ cone. These two lines are denoted in this paper as $l_{v d}$. Avoiding the obstacle by turning to the direction of one of the $l_{v d}$ will mean that $A_{o}$ is escaping as quick as possible to safety. However, because of the direct contact between $V O$ and $D I V, A_{o}$ will not experience the maintainmode, and goes directly to the restore after escaping $V O$, which indicates the oscillation problem of the VO-method ${ }^{15}$ will occur. The avoidance maneuver due to this feature needs to be further investigated by simulations.

\section{The Reachable Velocity Set}

In three dimensional space, a UAV can virtually turn in any direction to avoid. In accordance to the fan shaped $R V$ in the two dimensional definition, the $R V$ in three dimensions should be the surface revolution by the $\mathrm{X}$-axis of $A_{o}$, such as shown in Figure 6. This spherical-shell shape, however, is using the assumption that there is no gravity effect that makes the shape unsymmetrical.

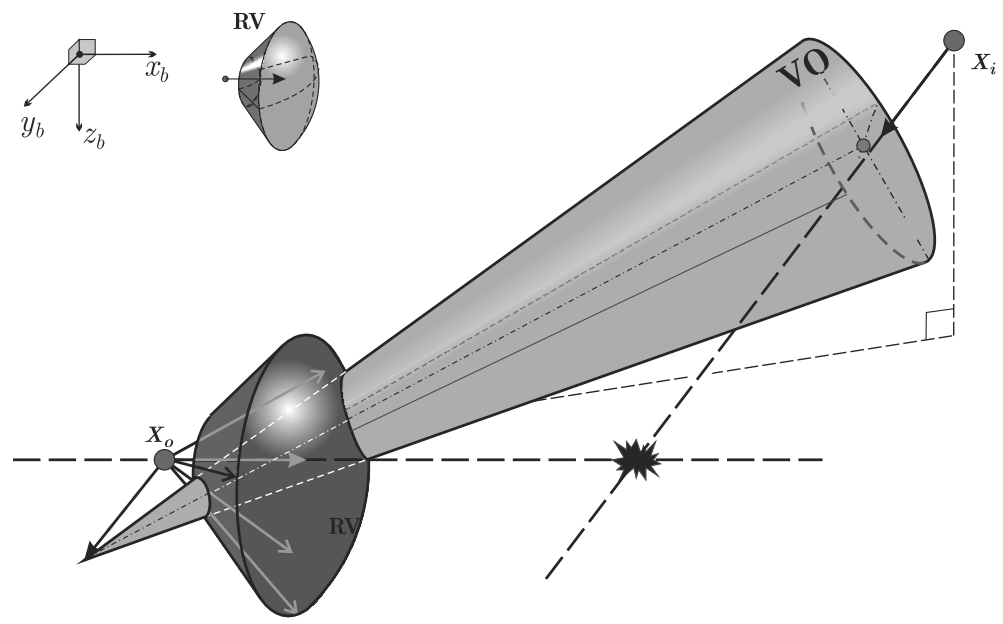

Figure 6. Reachable Velocity set, $R V$, definition in Three-dimensional space. The volume shape of $R V$ is the result of surface-revolution of the two-dimensional fan-shape $R V$, along the $\mathbf{X}$-axis.

If the strategy of avoidance remains the same, then the UAV needs to find the closest intersection point of the spherical shell $R V$ with the $V O$ cone. This model, however, is difficult to make sense of. This paper takes a simpler approach to assess and represent the interaction between the $V O$ set and the $R V$ set, by defining the avoidance planes, explained in the next section.

\section{The Avoidance Planes}

The direct three-dimensional setup for the Velocity Obstacle method, explained in the previous section, is complex and thus eliminates the purpose of the method to aid in the situational awareness. Finding a good strategy hence becomes difficult. For instance, if the ownship has a reachable velocity set like the case in figure 6 , then the intersection derivation from two volumes is required, i.e., between the set of $R V$ and the $V O$-cone. Defining the diverging set, the $R V$ set, and all the important intersection between them, are also difficult to illustrate. Therefore, this research introduces the avoidance planes, $P_{\phi}$, as tools to logically 
describe the three-dimensional case into separate two-dimensional setups, and provides a way to generate an avoidance strategy. This section will explain thoroughly their derivation and important parameters it has.

The avoidance plane is defined as any plane in which lies $A_{o}$ 's position, $X_{o}$, and velocity $V_{o}$. A UAV can therefore choose one of the planes in which it conducts the avoidance maneuver. Every set in the three dimensional Velocity Obstacle Method can be viewed as their cross-section area in each plane. By comparing between the avoidance planes, the own-ship $A_{o}$ can choose the best avoidance plane and make an optimal avoidance.

Ref 7 presents a similar method of three-dimensional case breakdown, but concentrated for two avoidance planes, which are the lateral plane (XY-Plane) and the longitudinal plane (XZ-Plane). ${ }^{7}$ The method of torus $^{16}$ for avoidance also resembles the method with an extensive calculation.

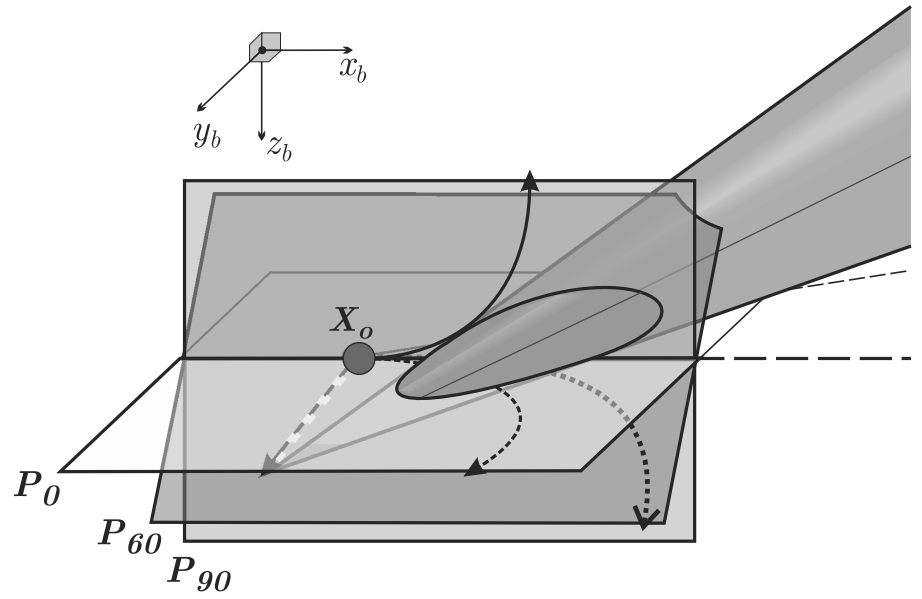

Figure 7. The avoidance plane. $A_{o}$ can choose its avoidance maneuver by moving in one selected plane, e.g. $P_{0}, P_{60}$, or $P_{90}$.

Consider a simple case of a three dimensional encounter as shown in Figure 8. The figure shows the structure of the velocity obstacle cone in three dimensional space, for the case where $A_{o}$ 's position and velocity are $\left[\begin{array}{lll}0 & 0 & 0\end{array}\right]^{T}$ meters and $\left[\begin{array}{lll}5 & 0 & 0\end{array}\right]^{T}$ meters/second, with an obstacle encountering from the left-upper side, whose global positions are $\left[\begin{array}{lll}150 & 50 & 50 \sqrt{2}\end{array}\right]^{T}$ meters, heading at the global direction of $\left[\begin{array}{lll}0 & -45 & 135\end{array}\right]^{T}$ degrees.

Figure 8 also shows the VO-cone as it is going through the $X Y$ and $X Z$-plane of the $A_{o}$ body frame of reference. The cross section on the plane is an ellipse for both $P_{0}$ and $P_{90}$. In fact, since the $V O$ is a right cone, for all avoidance plane, the $V O$ cross section will be a conic-section, i.e., circle, ellipse, parabola, or hyperbola. The two-dimensional triangular shape of $V O$ set is actually a special case of conic-section, that occurs when the $V O$ cone apex, or the obstacle velocity $V_{i}$ lies on the avoidance plane. There exist only one avoidance plane that reduce the three-dimensional case into the two-dimensional. In the next subsections, the definition of the $V O, D I V$ and the $R V$, and in an avoidance plane $P_{\phi}$ is elaborated.

\section{A. Velocity Obstacle Set on an Avoidance Plane}

In order to describe the conic-section in every avoidance plane $P_{\phi}$, the $V O$ cone is first described mathematically in the three-dimensional space by its apex $A_{v o}$, its right-base circle perimeter, $B_{v o}$, and the center of the base, $C_{v o}$. These parameters are expressed using parametric equations in Euclidean space, as presented in equation (6) until (9). The derivation is conducted with the definition of the position of the own-ship according to the spherical coordinates based on the own-ship body axis as the frame of reference, $X Y Z_{b}$, as shown in Figure 4-a.

$$
A_{v o}=\left[\begin{array}{l}
x_{A} \\
y_{A} \\
z_{A}
\end{array}\right]=V_{i}
$$




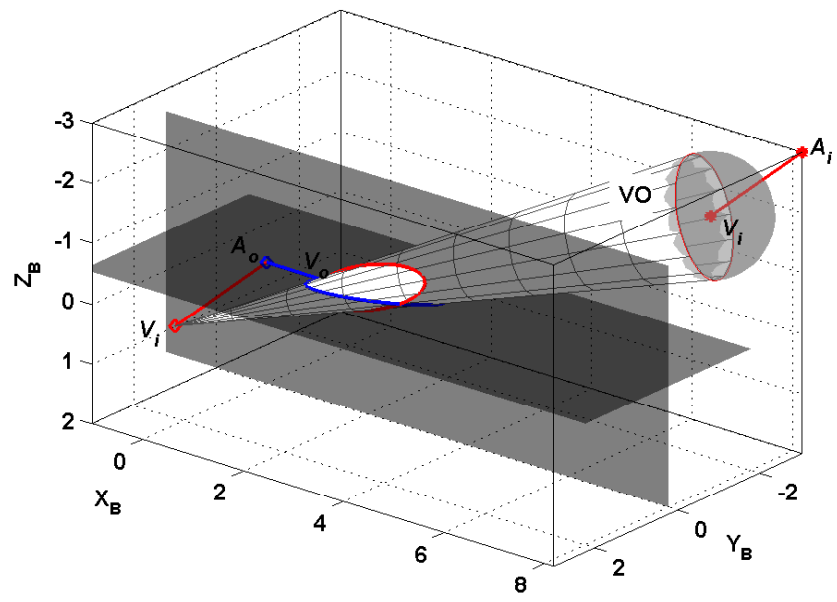

Figure 8. Example case of a three-dimensional encounter. The Velocity cone is shown, with apex on the $V_{i}$ vector, crossing through the $\mathrm{XY}$ and $\mathrm{XZ}$-plane of $A_{o}$ body frame of reference.

$$
\begin{gathered}
B_{v o}=\left[\begin{array}{c}
x_{B} \\
y_{B} \\
z_{B}
\end{array}\right]=R_{\theta_{e l} \mid \theta_{a z}}\left[\begin{array}{c}
d_{v o} \\
r_{v o} \cos t \\
r_{v o} \sin t
\end{array}\right]+V_{i}, \quad 0 \leq t \leq 2 \pi \\
C_{v o}=\left[\begin{array}{c}
x_{C} \\
y_{C} \\
z_{C}
\end{array}\right]=R_{\theta_{e l} \mid \theta_{a z}}\left[\begin{array}{c}
d_{v o} \\
0 \\
0
\end{array}\right]+V_{i},
\end{gathered}
$$

where,

$$
R_{\theta_{e l} \mid \theta_{a z}}=\left[\begin{array}{ccc}
\cos \theta_{a z} \cos \theta_{e l} & \sin \theta_{a z} & -\cos \theta_{a z} \sin \theta_{e l} \\
-\sin \theta_{a z} \cos \theta_{e l} & \cos \theta_{a z} & \sin \theta_{a z} \sin \theta_{e l} \\
\sin \theta_{e l} & 0 & \cos \theta_{e l}
\end{array}\right]
$$

Every generating-line, or generatrix, of the $V O$ cone that connects the cone apex with the base circle are expressed in equation (10). the $V O$ cone symmetric, on the other hand, is expressed in equation (11).

$$
\begin{gathered}
G_{v o}=\left[\begin{array}{l}
x_{g}(t) \\
y_{g}(t) \\
z_{g}(t)
\end{array}\right]=\left(B_{v o}-V_{i}\right) t_{g}+V_{i}, \quad 0 \leq t_{g} \leq 1 \\
H_{v o}=\left[\begin{array}{l}
x_{h} \\
y_{h} \\
z_{h}
\end{array}\right]=\left(C_{v o}-V_{i}\right) t_{h}+V_{i}, \quad 0 \leq t_{h} \leq 1
\end{gathered}
$$

Using equation (10), the conic-section on the xy-body axis, or $P_{0}$, in particular, can be derived by assigning $z_{g}=0$. The parametric $t_{g}$ value in equation (10) thus can be derived, and the conic-section for $P_{0}$ can be expressed as in equation (12).

$$
V O_{P_{0}}=\left[\begin{array}{c}
x_{v o} \\
y_{v o}
\end{array}\right]_{P_{0}}=\left[\begin{array}{c}
-\left(x_{B}-x_{A}\right) \frac{z_{A}}{\left(z_{B}-z_{A}\right)}+x_{A} \\
-\left(y_{B}-y_{A}\right) \frac{z_{A}}{\left(z_{B}-z_{A}\right)}+y_{A}
\end{array}\right]
$$

The equation for the $V O$ conic-section in any arbitrary avoidance plane $P$ can be found by virtually rotating the cone around the $\mathrm{X}$-axis of $A_{o}$, and use equation (10) and (12) again. The virtual rotation of thw $V O$ cone, corresponds with the avoidance plane of $P_{\phi_{p}}$, expressed in equation (13). 


$$
A_{v o}^{\phi}=\left[\begin{array}{c}
x_{A}^{\phi} \\
y_{A}^{\phi} \\
z_{A}^{\phi}
\end{array}\right]=R_{P_{\phi}} A_{v o}, \quad B_{v o}^{\phi}(t)=\left[\begin{array}{c}
x_{B}^{\phi}(t) \\
y_{B}^{\phi}(t) \\
z_{B}^{\phi}(t)
\end{array}\right]=R_{P_{\phi}} B_{v o}(t)
$$

where,

$$
R_{P_{\phi}}=\left[\begin{array}{ccc}
1 & 0 & 0 \\
0 & \cos \phi & \sin \phi \\
0 & -\sin \phi & \cos \phi
\end{array}\right]
$$

Now equation (12) can be rewritten for any avoidance plane $P_{\phi}$ as expressed in equation (15).

$$
V O_{P_{\phi}}=\left[\begin{array}{l}
x_{v o}(t) \\
y_{v o}(t)
\end{array}\right]_{P_{\phi}}=\left[\begin{array}{c}
-\left(x_{B}^{\phi}(t)-x_{A}^{\phi}\right) \frac{z_{A}^{\phi}}{\left(z_{B}^{\phi}(t)-z_{A}^{\phi}\right)}+x_{A}^{\phi} \\
-\left(y_{B}^{\phi}(t)-y_{A}^{\phi}\right) \frac{z_{A}^{\phi}}{\left(z_{B}^{\phi}(t)-z_{A}^{\phi}\right)}+y_{A}^{\phi}
\end{array}\right],
$$

where

$$
z_{B}^{\phi} \neq z_{A}^{\phi}, \quad 0 \leq t \leq 2 \pi
$$

The point where the cone symmetry axis intersect with $P_{\phi}$, can be derived in the same manner, which result in equation (17).

$$
H_{P_{\phi}}=\left[\begin{array}{c}
x_{h} \\
y_{h}
\end{array}\right]_{P_{\phi}}=\left[\begin{array}{c}
-\left(x_{C}^{\phi}-x_{A}^{\phi}\right) \frac{z_{A}^{\phi}}{\left(z_{C}^{\phi}-z_{A}^{\phi}\right)}+x_{A}^{\phi} \\
-\left(y_{C}^{\phi}-y_{A}^{\phi}\right) \frac{z_{A}^{\phi}}{\left(z_{C}^{\phi}-z_{A}^{\phi}\right)}+y_{A}^{\phi}
\end{array}\right],
$$

Figure 9 shows twelve examples of conic sections of the $V O$ cone generated in the example of Figure 8, illustrated at $A_{o}$ 's body axis as the frame of reference. The conic-sections of $V O$ are shown by the shaded elliptical areas. The thick line on the x-axes represents $V_{o}$, while the thick gray line represents the projection of $V_{i}$ on each avoidance plane $P_{p h i}$. The star inside each area represent the intersection point of the symmetry axis, the $C_{v o} \phi$. The dashed line represents the projection of $V O$ cone axis of symmetry. Due to the cone symmetry, this line will always coincides with the major axis of every $V O$ conic-section.

Equation (15) works well in defining an ellipse area. For parabolic and hyperbolic area, however, another consideration is required. In both case, not all generating line in the finite cone of $V O_{P}$ will intersect with the avoidance plane $P_{\phi}$. This is the singular condition from the equation, when $z_{B}^{\phi}(t)=z_{A}^{\phi}(t)$. To avoid the problem, the $V O_{P}$ is determined as a polygon that connects the points resulting from equation (15), only when $0 \leq t_{g} \leq 1$. In other words, only consider the finite $V O$ cone. With this setup, the area on the 'other cone' in the hyperbolic result is also removed as well.

A special degenerate case is shown in $P_{45}$, in which $V_{i}$, or $A_{v o}^{\phi}$ is included in the avoidance plane. In this condition, the conic section taking a triangular shape, where all generating line from $A_{v o}$ to $B_{v o}$ crosses the avoidance plane $P$ in the same point, i.e., the $V_{i}$, except for two line. The $V O_{P}$ triangle area is limited by this two line, expanding until the $d_{v o}$. Hence, generating the $V O_{P}$ using the parametric equation (15) is impossible without defining these two line. The point of these line on the base circle $B_{v o}^{\phi}$ can be derived using the singular condition of equation (15), that is, when $z_{B}^{\phi}(t)=z_{A}^{\phi}(t)=0$.

The case of triangular shape is one from three degenerate cases that can occur when the cone apex, $A_{v o}$, is included in the $P_{\phi}$. If the condition of $z_{B}^{\phi}(t)=z_{A}^{\phi}(t)=0$ only result in one point on the cone base, the $V O_{P}$ will be a single line. If instead there are no solution found, then $V O_{P}$ will be a single point, that is the cone apex, $A_{v o}$ itself. It is interesting to observe that on every case of encounter there always exist at least one avoidance plane $P_{\phi}$ that creates this degenerate case. If the vector of $V_{o}$ is parallel with $V_{i}$, then every case of $V O_{P}$ is degenerate.

In general, using the acute dihedral angle between the avoidance plane, $P_{\phi}$ and the cone base-circle $B_{v o}$, the type of shape of the $V O_{P}$ can be defined. This angle is denoted as $\theta_{P_{\phi}}$ and derived in the following equation (18).

$$
\theta_{P_{\phi}}=\arccos \left(\frac{z_{C}^{\phi}-z_{A}^{\phi}}{d_{v o}}\right)
$$



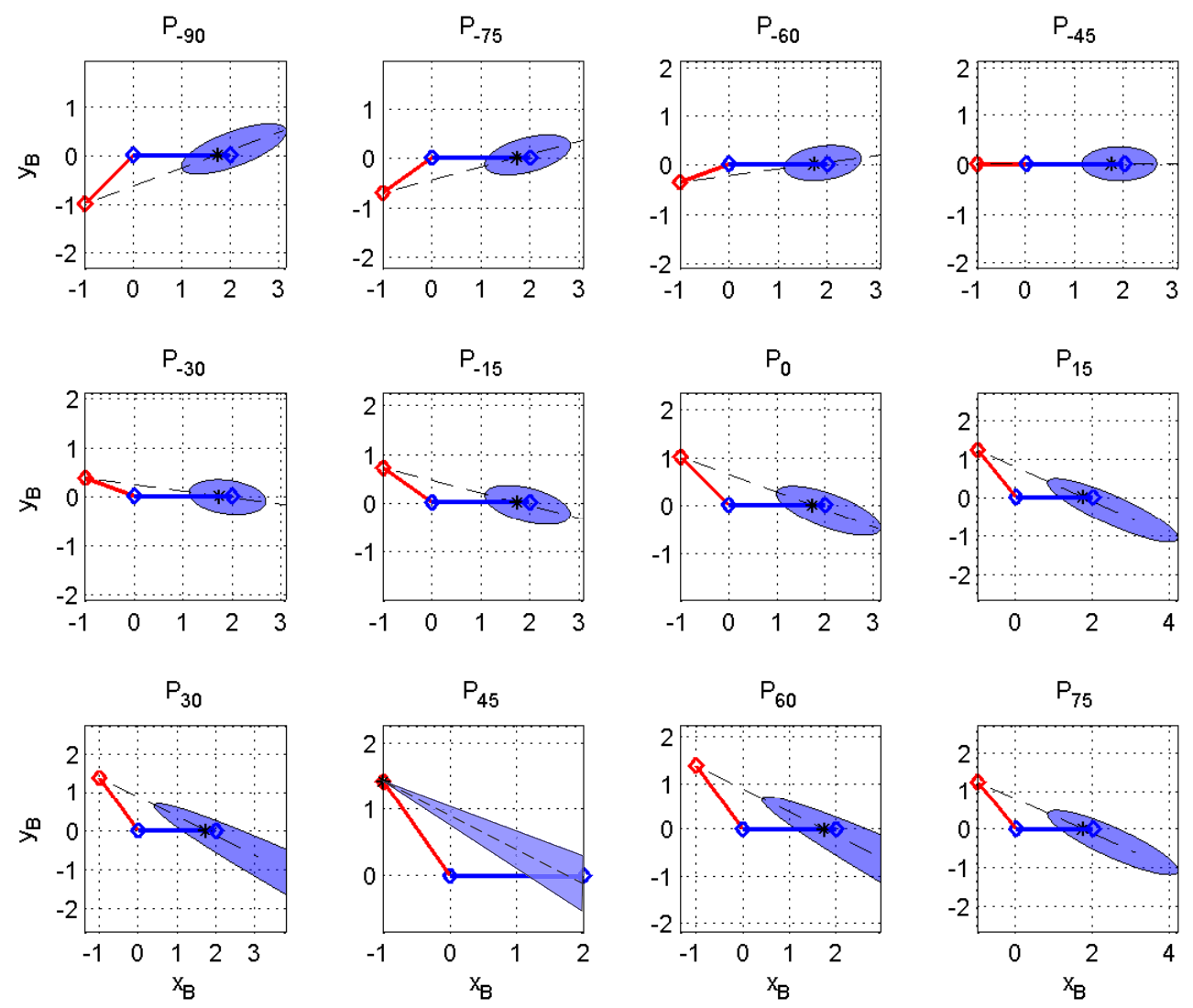

Figure 9. Conic-sections of the $V O$ cone on several avoidance-planes. The thick blue line on $\mathrm{X}$-Axis represent $V_{o}$, while the thick gray line is the $V_{i}$ projection on the avoidance plane. The dashed line from $V_{i}$ is the projection of $V O$ 's cone axis of symmetry.

Together with the degenerate cases, the types of area of the $V O_{P}$ are summarized in the following list.

1. if $\theta_{P_{\phi}}=0$, then $V O_{P}$ is a circle, if also $A_{v o} \in P_{\phi}$, then $V O_{P}$ is a single point.

2. if $\theta_{P_{\phi}}<\pi / 2-\theta_{v o}$, then $V O_{P}$ is an ellipse, if also $A_{v o} \in P_{\phi}$, then $V O_{P}$ is a single point.

3. if $\theta_{P_{\phi}}=\pi / 2-\theta_{v o}$, then $V O_{P}$ is a parabolic area, if also $A_{v o} \in P_{\phi}$, then $V O_{P}$ is a straight line.

4. if $\theta_{P_{\phi}}>\pi / 2-\theta_{v o}$ and $P_{\phi}$ does not cut through the $V O$-cone axes, then $V O_{P}$ is a hyperbolic area, if also $A_{v o} \in P_{\phi}$, then $V O_{P}$ is a triangle.

Equation (15) can be used to further determine the property of the $V O_{P_{\phi}}$ set, treating the set as a polygon. For the Velocity Obstacle Method, the most important property is whether the $V_{o}$ is included in the $V O_{P}$ set or not. Equation (4) still hold in this context. Another way can also be used to keep the focus on an avoidance plane, that is, by finding the intersection of the $V O_{P}$ with the x-axis. If there are two intersection point, in which only one of them has value less then $V_{o}$, then the $V_{o}$ is inside the $V O_{P}$.

Two other important properties are the set area, $S_{V O_{P}}$, and the closest point of $V_{a v o}$. The area is related to the risk the ownship must consider in its avoidance on the chosen avoidance-plane $P_{\phi}$. The smaller the area is, the more option of $V_{\text {avo }}$ the ownship has. Figure 9 shows that $P_{-45}$ has the smallest area, while $P_{45}$, as a triangle, has the largest. The closest point for avoidance, $V_{a v o}$, however, is not only depends on the area, but on the type of shape and the area axis attitude as well. The area for a polygon with $n$-vertices can be defined using equation (19). The closest vertex of the polygon from the current velocity, on the other hand, is defined as the closest selection for an avoidance velocity, $V_{\text {avo }}^{\min }$, as expressed in equation (20). 


$$
\begin{gathered}
S_{V O_{P}}=\frac{1}{2}\left|\sum_{t=0}^{n-1}\left(x_{v o}(t) y_{v o}(t+1)-x_{v o}(t+1) y_{v o}(t)\right)\right| \\
V_{\text {avo }}^{\min }=\left\{V_{a v o}(t) \mid t=\arg \min \sqrt{\left.\left(x_{v o}(t)-V_{o}\right)^{2}+\left(y_{v o}(t)\right)^{2}\right)}\right\}
\end{gathered}
$$

where,

$$
t=\left\{0, \frac{2 \pi}{n}, \frac{4 \pi}{n}, \ldots, \frac{2 t \pi}{n}, 2 \pi\right\}
$$

\section{B. Diverging Zone on an Avoidance Plane}

Similar in the direct three dimensional $V O$ method, the diverging zone $D I V$ on an avoidance plane can be defined by moving the set of $V O_{P}$ along the $V_{i}$, as shown in figure 10. It can be observed that the edges of the zone are the tangential lines of the $V O_{P}$ from the origin, or $A_{o}$ current position.
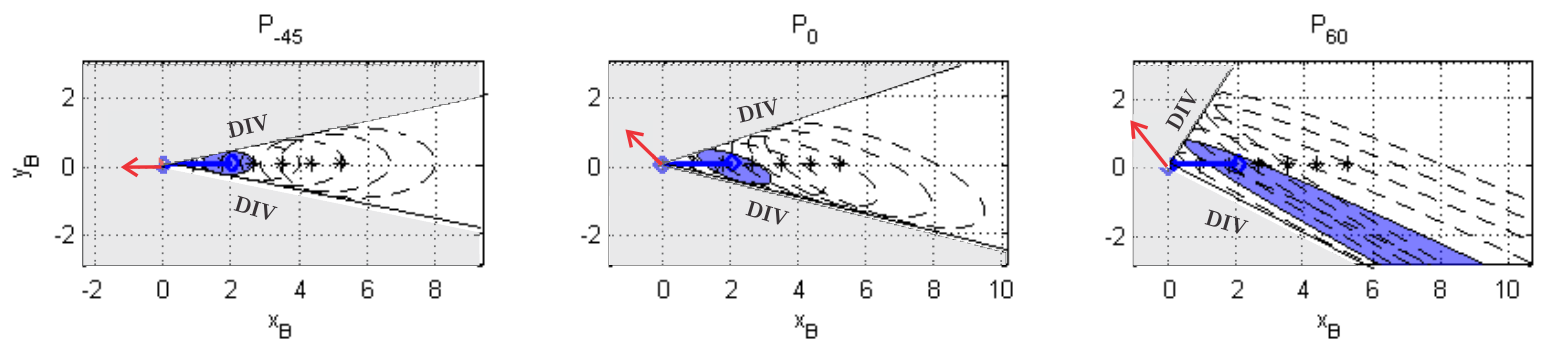

Figure 10. Diverging zone set, $D I V$, in three different avoidance-planes.

Using the $V O_{P}$ as a polygon, those tangential lines are the two lines that pass through the origin and a vertex on the set that have the maximum or minimum value of gradient. The inclusion of $V_{o}$ in the $D I V_{P}$ set thus can be determined by checking if for each vertex, the criteria in equation (22) are fulfilled.

$$
\forall t: \frac{y_{v o}(t)}{x_{v o}(t)}>0 \quad \vee \quad \forall t: \frac{y_{v o}(t)}{x_{v o}(t)}<0
$$

The diverging zone became more dominant in three dimensional cases. This means that restore-mode is easier to experience, especially with a high value of turning rate. Based on equation (22), for each avoidance plane, $V O_{P}$ and $D I V_{P}$ will always have two specific point of intersection, denoted as $l_{v d}$, which is also the two points that determined the $D I V_{P}$ edges. This is true except for the degenerate cases.

\section{Reachable Velocity Set on an Avoidance Plane}

The $R V$ can be defined in each avoidance plane as an area, which will simply revert back to its two dimensional fan-shaped area. Figure 11 shows the cross-sectional area of $R V$ on two examples of avoidance planes, $P_{0}$, and $P_{-90}$, which corresponds to the ownship's $X Y$, or longitudinal, and $X Z$, or lateral, -planes, respectively. Again, this is for the case shown in Figure 8.

$R V$ definition neglects the effect of gravity, and therefore the $R V$ on $P_{90}$ is symmetrical, unlike the real longitudinal flight envelope of an aircraft, as shown in Ref.7. If the own-ship only considers these two planes, then the best avoidance for the case is either to turn right, or to decent, as they have the closest escape route from the $V O$. However, if the gravity effect is included, the easiest way to make avoidance is probably to descent, or to turn right on the $P_{-90}$. If $A_{o}$ also considers how to escape to the $D I V$ zone as soon as possible, the avoidance might be different. In this case, the own-ship needs to change its velocity directly to one of the $l_{v d}$ points. With this in consideration, the opposite maneuvers to turn left or climb, are the better solution. These maneuvers make sense, since they go in the opposite direction of the obstacle.

Figure 11-a and -b also show the complete form of the $V O$ sets on an example of avoidance-planes. The figure shows the entire feature of the selected avoidance-planes, including the representation of the main 


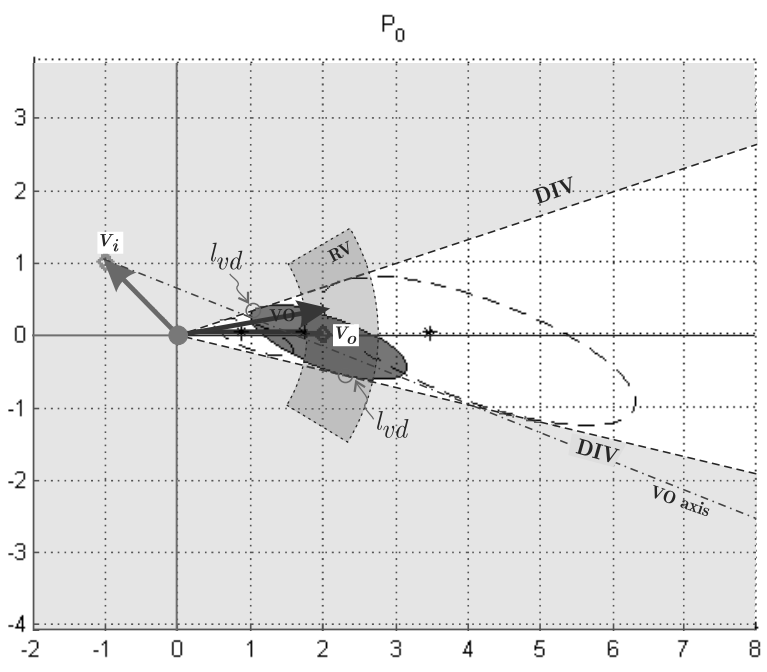

(a)

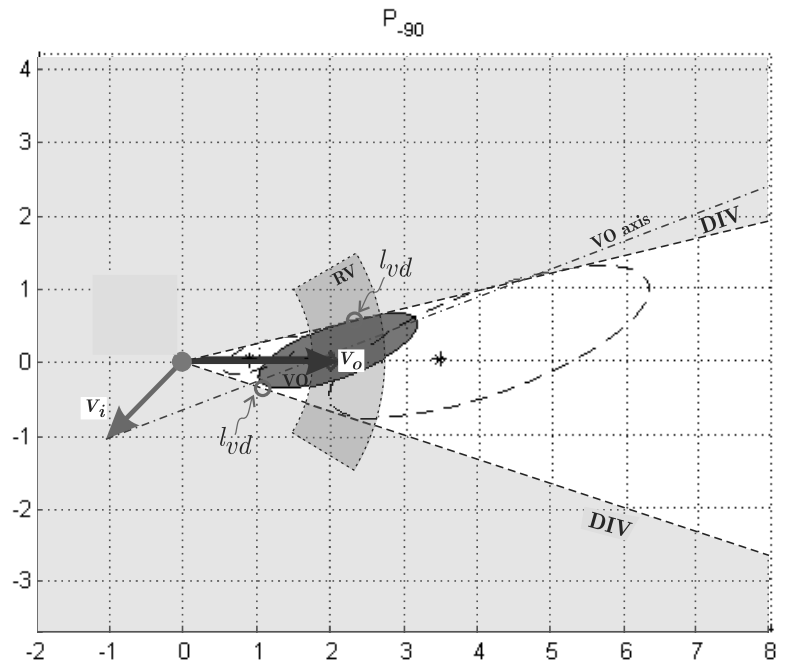

(b)

Figure 11. Complete form of two example of the Avoidance Plane, for encounter case shown in Figure 8. (a) the $P_{0}$, represent the $X Y$ or longitudinal- plane, and (b) $P_{-90}$, which corresponds to $A_{o} \mathrm{~s} X Z$, or lateral-planes of $A_{o}$ body axis

sets, $V O, D I V$ and $R V$, along with the $V O$ cone axis of symmetry, and the intersection point of $V O-D I V$ set, $l_{v d}$. The use of these features needs to be elaborated further, especially to determine its dynamics with the progression of the encounter.

\section{Implementation}

To evaluate the method presented in this paper, a computer simulation program has been developed. The simulated vehicles are assumed to exchange flight-data among each other, which includes the position, heading, and speed. The exchanges are assumed to be perfect without any losses or delays. The propagation of the avoidance maneuver in the simulation is shown using time-capture frames. Each vehicle involved is depicted as a point mass in the 3 -dimensional space, set to have 5 meters/second of speed. The protected-zone of all dynamic (non-maneuvering) obstacles are depicted as spheres with radius of 10 meters. The own-ship starts its avoidance from $d_{\text {avo }}=40 \mathrm{~m}$, with a constant turning-rate magnitude of 15 degrees/second. A special control algorithm is given to the own-ship in order to point back to a far original goal (500 meter away), after the obstacle is cleared.

\section{A. Simulation 1: Two Vehicles Converging}

This is the simulation of the case used in the explanation of the three dimensional Velocity Obstacle, shown in Figure 8. Here, $A_{o}$ successfully avoids the protective zone $S_{p z}$ of the obstacle using tree choice of avoidance plane, $P_{0}, P_{45}$, and $P_{-90}$.

The own-ship in the simulation is able to avoid the obstacle, with the nearest distance of 10 meters, exactly at the Protected Zone sphere surface, as also shown in Figure 14. The three Avoidance planes are demonstrated able to avoid collision with the intruder. Which plane is the optimal avoidance, however, requires further extraction from the simulation result.

It should be note that work of avoidance using the Velocity Obstacle method ends on the highest point of each avoidance, where the course is considered as clear. The pointing back to the original goal (restore) is the work of a control system separately from the 3-dimensional Velocity Obstacle. 


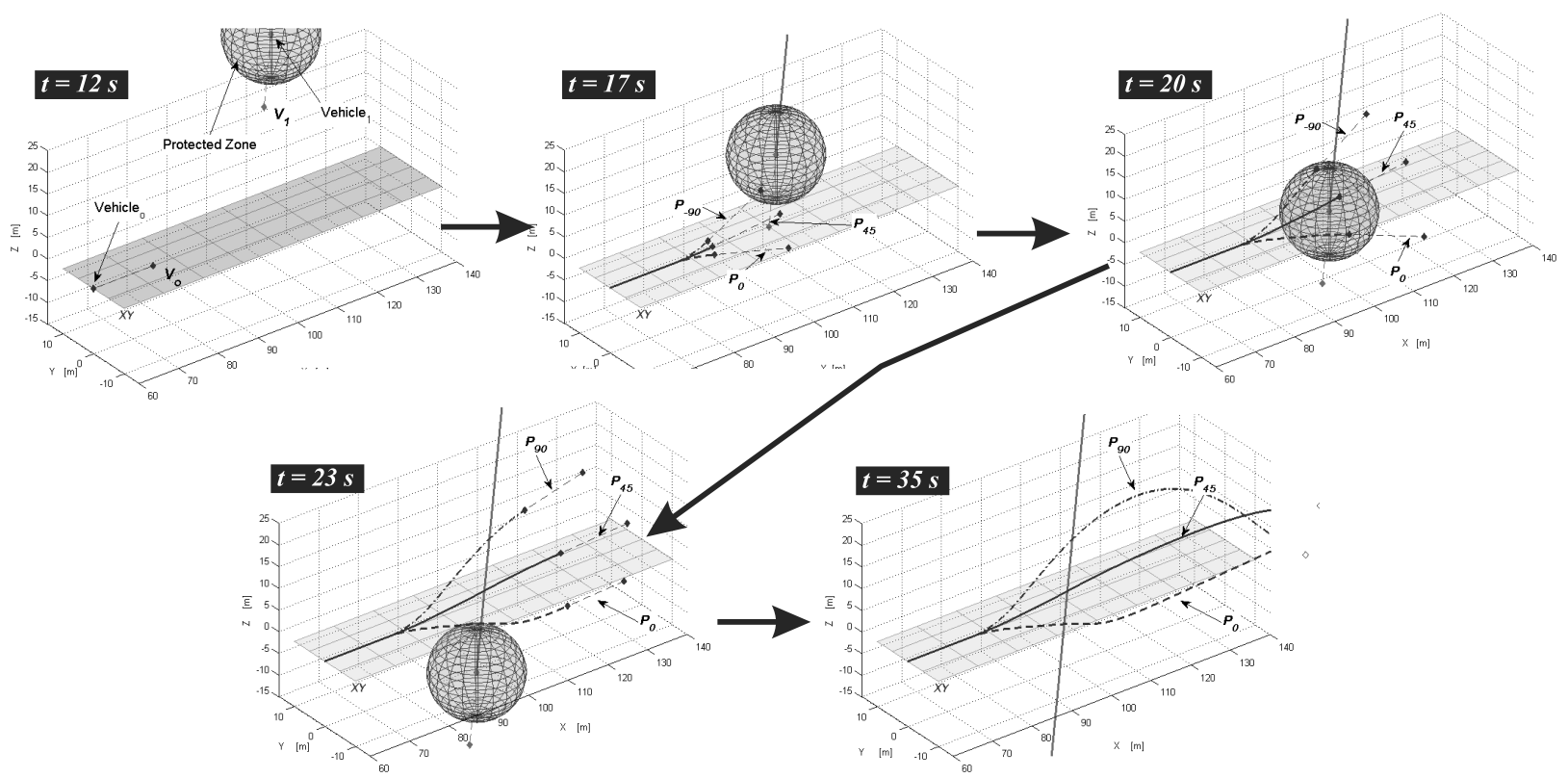

Figure 12. Simulation-1: The case shown in Figure 8. Three choice of avoidance plane is given, $P_{0}, P_{45}$, and $P_{-90}$

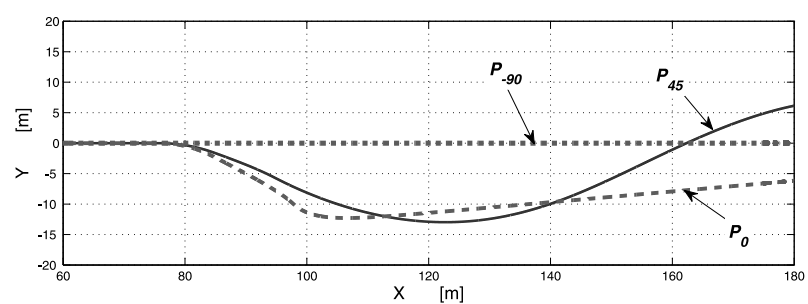

(a)

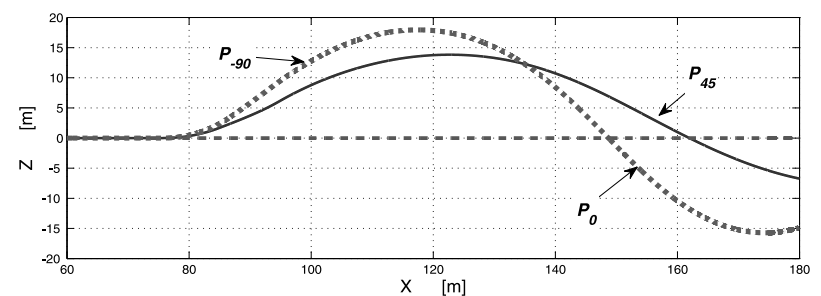

(b)

Figure 13. Simulation-1: (a) Top-view and (d) Side View of the own-ship flight-path in its avoidance, using three choice of avoidance plane, $P_{0}, P_{45}$, and $P_{-90}$

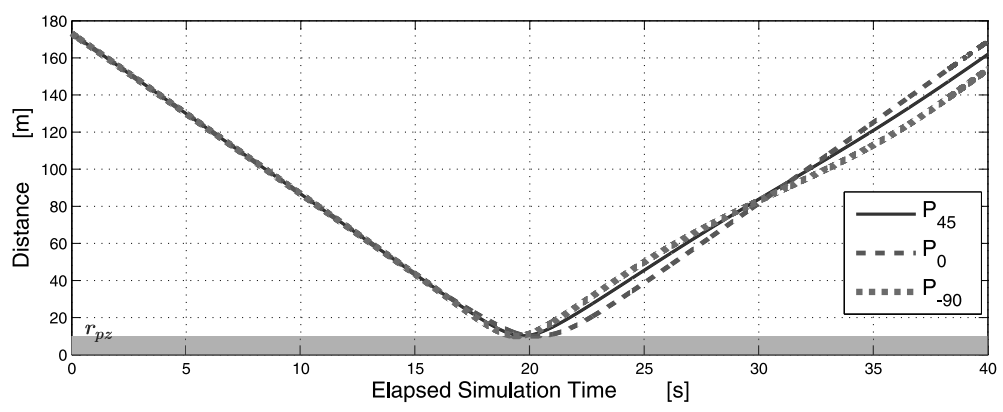

Figure 14. Simulation-1: Distance through the simulation time, with three choice of avoidance plane $P_{0}, P_{45}$, and $P_{-90}$

\section{B. Simulation 2: Multiple Conflicts}

Figure 15 shows the simulation of the case of multiple 3-dimensional conflicts, avoided by the ownship using three different avoidance plane. Here, three obstacle, $V_{1}, V_{2}$ and $V_{3}$ are set to collide with the own-ship along its path. The own-ship avoid by turning on an avoidance plane that produce the smallest conic-sections, i.e. 
$P_{45}, P_{0}$, and $P_{-90}$, respectively. The avoidance is conducted based on the distance priority,

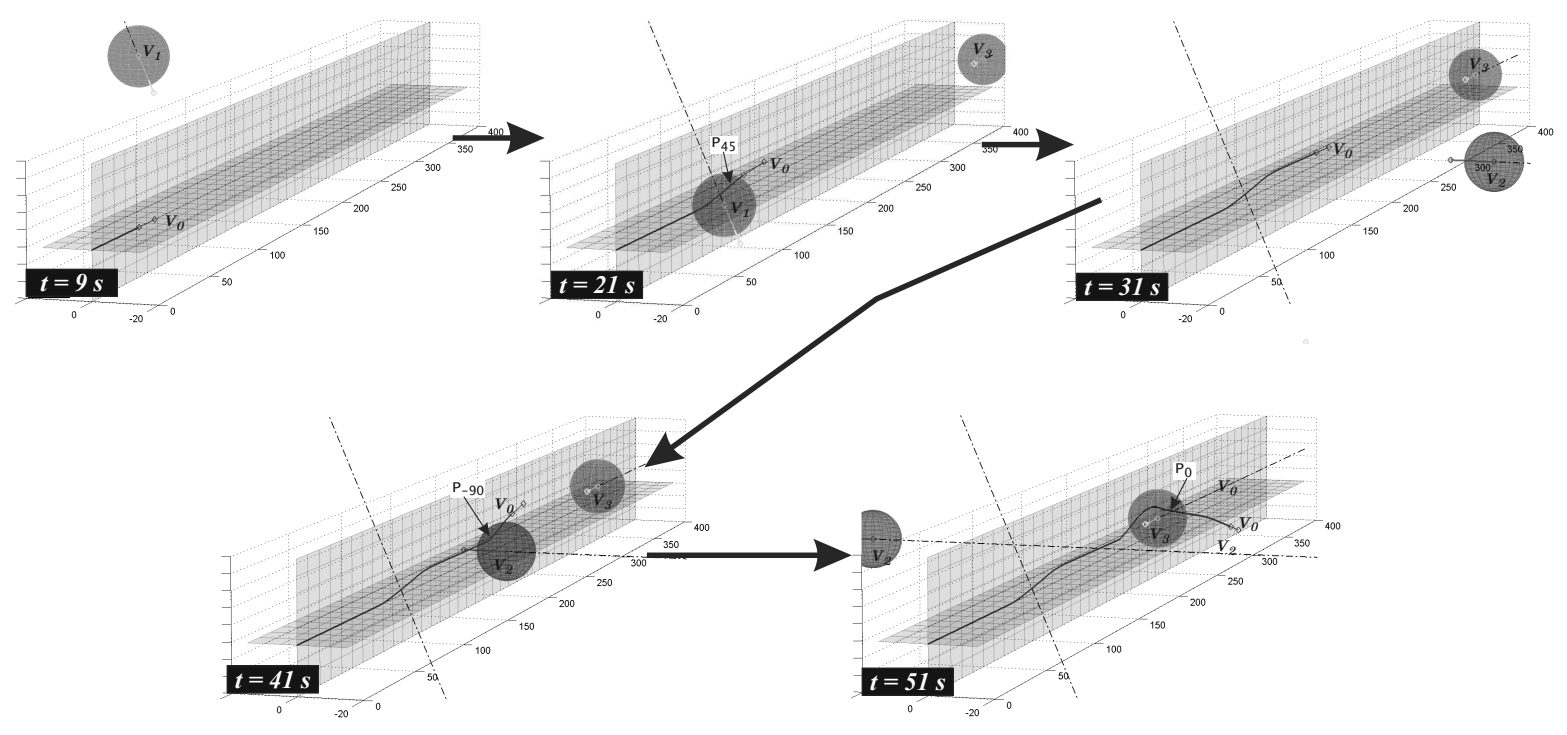

Figure 15. Simulation-2: Multiple 3-dimensional conflicts, avoided by the ownship using three different avoidance plane, i.e. the smallest $V O$ conic section.

\section{Conclusion}

This paper focuses on the assessment of the Velocity Obstacle Method to autonomously resolve threedimensional conflicts for Unmanned Aerial Vehicles (UAVs). A novel technique to represent the threedimensional conflict in various avoidance-planes is introduced to help the avoidance decision making.

The use of avoidance-planes reveals many interactions between the conflict and the avoidance maneuver in three-dimensions. Choosing an avoidance plane on $P_{x y}$ or $P_{x z}$ will result in a pure turn or climb/descent, respectively. The risk value for each avoidance-plane can be defined by the area of the $V O$ conic-section in each avoidance-plane. An avoidance with highest risk is experienced whenever the vehicle chooses an avoidance plane on which lies the obstacle velocity vector $V_{i}$. The least-risk avoidance, on the other hand, occurs on the avoidance-plane that is perpendicular from the previous. The risk value, however, may not necessary be related to the avoidance-plane with the shortest path.

Simulations are conducted to assess the performance of the algorithm in a single or multiple conflict. Further analysis need to be conducted to determined the effectiveness and efficiency of the maneuver, related to the aim of deconflict maneuver.

The large domination of diverging areas in three-dimensional cases suggest that it is easier for a vehicle to avoid conflict, compared to the two dimensional case. On every avoidance-plane exists two points of $V O$ - DIV intersection, which represent the fastest escape route from $V O$. However, oscillation is expected to occur. The technique needs to be elaborated further, especially for dynamic changes of the avoidance-plane. The reciprocating cases are left for the future work as well.

\section{References}

\footnotetext{
${ }^{1}$ DeGarmo, M. T. and Nelson, G. M., "Prospective unmanned aerial vehicle operations in the future national airspace system," AIAA 4th Aviation Technology, Integration, and Operations Forum, ATIO, AIAA, 2004, pp. 172-179, AIAA 20046243, doi:10.2514/6.2004-6243.

${ }^{2}$ Jenie, Y. I., van Kampen, E., de Visser, C. C., and Chu, Q. P., "Selective Velocity Obstacle Method for Cooperative Autonomous Collision Avoidance System for UAVs," AIAA Guidance, Navigation, and Control Conference 2013, AIAA, Boston, MA, 2013, AIAA 2013-4627, doi:10.2514/6.2013-4627.

${ }^{3}$ Hoekstra, J. M., Ruigrok, R. C. J., and van Gent, R. N. H. W., "Free Flight in a Crowded Airspace ?" 3rd USA/Europe Air Traffic Management $R$ \& D Seminar - Proceedings, EUROCONTROL, Napoli, 2000.
} 
${ }^{4}$ Hoekstra, J. M., "Designing for Safety: the Free Flight Air Traffic Management concept," 2001.

${ }^{5}$ Barfield, F., "Autonomous collision avoidance. The technical requirements," National Aerospace and Electronics Conference (NAECON), 2000, pp. 808-813, doi:10.1109/NAECON.2000.894998.

${ }^{6}$ Ellerbroek, J., Visser, M., van Dam, S. B. J., Mulder, M., and van Paassen, M. M., "Design of an Airborne ThreeDimensional Separation Assistance Display," IEEE Transactions on Systems, Man, and Cybernetics, part A: Systems and Humans, Vol. 41, No. 6, 2011, pp. 863-875, doi:10.1109/TSMCA.2010.2093890.

${ }^{7}$ Ellerbroek, J., Brantegem, K. C. R., van Paassen, M. M., and Mulder, M., "Design of a Co-Planar Airborne Separation Display," IEEE Transactions on Human-Machine Systems, Vol. 43, No. 3, 2013, pp. 277-289, doi:10.1109/TSMC.2013.2242888.

${ }^{8}$ Fiorini, P. and Shiller, Z., "Motion Planning in Dynamic Environments Using Velocity Obstacles," The International Journal of Robotics Research, Vol. 17, No. 7, 1998, pp. 760-772, doi:10.1177/027836499801700706.

${ }^{9}$ Kluge, B. and Prassler, E., "Recursive Probabilistic Velocity Obstacles for Reflective Navigation," Field and Service Robotics, edited by S. Yuta, H. Asama, E. Prassler, T. Tsubouchi, and S. Thrun, Vol. 24 of Springer Tracts in Advanced Robotics, Springer Berlin Heidelberg, 2006, pp. 71-79, doi:10.1007/10991459_8.

${ }^{10}$ van der Berg, J., Lin, M., and Manocha, D., "Reciprocal Velocity Obstacles for Real-Time Multi-Agent Navigation," International Conference on Robotics and Automation, IEEE, Pasadena, CA, USA, 2008, doi:10.1109/ROBOT.2008.4543489.

${ }^{11}$ van Dam, S. B. J., Mulder, M., and Van Paassen, M., "Ecological Interface Design of a Tactical Airborne Separation Assistance Tool," Systems, Man and Cybernetics, Part A: Systems and Humans, IEEE Transactions on, Vol. 38, No. 6, Nov 2008, pp. 1221-1233, doi:10.1109/TSMCA.2008.2001069.

${ }^{12}$ Heylen, F. M., van, S. B. J., Mulder, M., and van Paassen, M. M., "Design and Evaluation of a Vertical Separation Assistance Display," AIAA Guidance, Navigation, and Control Conference and Exhibit, Honolulu (HI), 2008, AIAA 20086969, doi:10.2514/6.2008-6969.

${ }^{13}$ Snape, J., van den Berg, J., Guy, S., and Manocha, D., "The Hybrid Reciprocal Velocity Obstacle," Robotics, IEEE Transactions on, Vol. 27, No. 4, Aug 2011, pp. 696-706, doi:10.1109/TRO.2011.2120810.

${ }^{14}$ Jenie, Y. I., van Kampen, E.-J., and Remes, B., "Cooperative Autonomous Collision Avoidance System for Unmanned Aerial Vehicle," Advances in Aerospace Guidance, Navigation and Control, edited by Q. Chu, B. Mulder, D. Choukroun, E.-J. Kampen, C. Visser, and G. Looye, Springer Berlin Heidelberg, 2013, pp. 387-405, doi:10.1007/978-3-642-38253-6_24.

${ }^{15}$ Kluge, B., "Recursive Probabilistic Velocity Obstacles for Reflective Navigation," Proc. of 1st Int. Workshop on Advances in Service Robots., 2003.

${ }^{16}$ Hurley, R. D., Lind, R., and Kehoe, J. J., "A Torus Based Three Dimensional Motion Planning Model for Very Maneuverable Micro Air Vehicles," AIAA Guidance, Navigation, and Control Conference 2013, AIAA, Boston, MA, 2013, AIAA 2013-4938, doi:10.2514/6.2013-4938. 